\title{
Ultrasensitiver nicht-enzymatischer Glukosesensor auf der Basis eines nanostrukturierten 3D-Spongin-Atacamit-Komposits
}

\author{
Sedigheh Falahi, laroslav Petrenko, Hermann Ehrlich, Yvonne Joseph, Parvaneh Rahimi* \\ Instituts für Elektronik- und Sensormaterialien, Fakultät für Werkstoffwissenschaft und Werkstofftechnologie, \\ TU Bergakademie Freiberg, Freiberg/Deutschland \\ Kontakt: Parvaneh.Rahimi@esm.tu-freiberg.de
}

\section{Einleitung}

Diabetes mellitus ist ein chronisch fortschreitendes Stoffwechselsyndrom und verursacht viele weitere Gesundheitsprobleme. Auf der Grundlage der Daten der Weltgesundheitsorganisation (WHO) und der International Diabetes Federation wird erwartet, dass sich die Zahl der Diabetiker bis zum Jahr 2045 verdoppeln wird [1]. Daher kann eine sorgfältige Behandlung von Diabetes akute Probleme verhindern und das Fortschreiten verzögern. Unter den verschiedenen Verfahren zum Nachweis von Glukose haben elektrochemische (Bio-)Sensoren aufgrund ihrer Vorteile, wie z. B. geringe Kosten, einfache und leichte Bedienung, schnelles Ansprechen und Point-of-Care-Nachweisfähigkeit, große Aufmerksamkeit auf sich gezogen [2]. In den letzten Jahren besteht ein großes Interesse an der Entwicklung enzymfreier elektrochemischer Sensor-Assays, die auf verschiedenen biokompatiblen Metall-Nanostrukturen und deren Oxiden als Katalysatoren für die Elektrooxidation von Glukose basieren. Nicht-enzymatische Glukosesensoren überwinden die Nachteile enzymatischer Glukosesensoren wie schlechte Stabilität/Reproduzierbarkeit, Abbau der Enzymaktivität, komplizierter Immobilisierungsprozess und hohe Kosten [3,4].

Es ist auffällig, dass das Sensormaterial als Elektrokatalysator eine Schlüsselrolle bei der Entwicklung nicht-enzymatischer Glukosesensoren spielt. Daher ist die Entwicklung effizienter Elektrodenmaterialien mit hervorragender katalytischer Aktivität, großer Oberfläche, wünschenswerter Morphologie, Größe, Form und Komposition ein wichtiger Faktor für die Konstruktion nicht-enzymatischer Glukosesensoren [2,5]. Nanomaterialien aus Kohlenstoff (wie Graphen, Kohlenstoff-Nanoröhren) [6,7], Metallen (Au, Zn, Ni, $\mathrm{Cu}, \mathrm{Pd})$ [8,9-11], Metalloxiden ( $\mathrm{ZnO}, \mathrm{CuO})[12,13]$, Legierungen $[14,15]$ (PtPb, AuNi) und Kompositen wurden ausgiebig als Elektrokatalysatoren für die nicht-enzymatische Glukosesensorik genutzt. Nanomaterialien/Kompositen auf $\mathrm{Cu}$-Basis wurden als vielversprechende Kandidaten für die Entwicklung elektrochemischer, nicht-enzymatischer Glukosesensoren anerkannt, da sie kostengünstig sind, eine hervorragende katalytische Aktivität aufweisen, stabil, ungiftig und biokompatibel sind. Unerwünschte elektronische Strukturen von Nanomaterialien/Kompositen auf Cu-Basis mit begrenzter Oberfläche können jedoch zu einem Verlust aktiver Stellen für die Adsorption und Reaktion von Glukose führen, was die elektro-katalytische Kinetik verringert [16]. Um diese Herausforderung zu überwinden und die effektive Oberfläche zu verbessern, können Cu-Nanostrukturen mit zweidimensionalen (2D) Materialien (z.B. Graphen) und der
Herstellung von Nanokomplexen $[16,17]$ oder mit dreidimensionalen (3D) Materialien (z.B. Schäumen) und der Entwicklung von nanoporösen Kompositen integriert werden. Im Vergleich zu den verschiedenen regulierten Nanostrukturen sind nanoporöse 3D-Materialien aufgrund ihrer größeren Oberfläche und ihrer einzigartigen elektrochemischen Eigenschaften in letzter Zeit zu attraktiven Elektrokatalysatoren geworden. Die Herstellung solcher mikro- oder nanoporöser Materialien ist jedoch komplex und teuer, um eine kostengünstige Plattform für die Glukose-sensorik zu entwickeln.

Natürliche Polymere bieten eine große Vielfalt an Matrizen für die Entwicklung von biokompatiblen 3D-Kompositen für Sensoranwendungen. In letzter Zeit hat das erneuerbare proteinartige Biopolymer Spongin, das die Hauptstruktur-komponente der Skelettfasern von Badeschwämmen (De-mospongiae: Porifera) darstellt, die Aufmerksamkeit der Gemeinschaft der Biomaterialien und Biomimetiker auf sich gezogen $[18,19]$. Unter bestimmten Bedingungen kann Spongin mit verschiedenen Metallionen wie $\mathrm{Fe}, \mathrm{Hg}$ und $\mathrm{Cu}$ reagieren und erhält auf diese Weise neue Funktionen, ohne seine ursprüngliche Porosität und einzigartige 3DMikro- und Makroarchitektur zu verlieren [20].

Hierin wurde zum ersten Mal ein neuer enzymfreier Sensor für die direkte Messung von Glukose hergestellt und elektrochemisch charakterisiert, der auf der Verwendung eines 3D-Spongin-Atacamit-Komposits (Sp. A) als biokompatibler Katalysator basiert (Abb. 1).

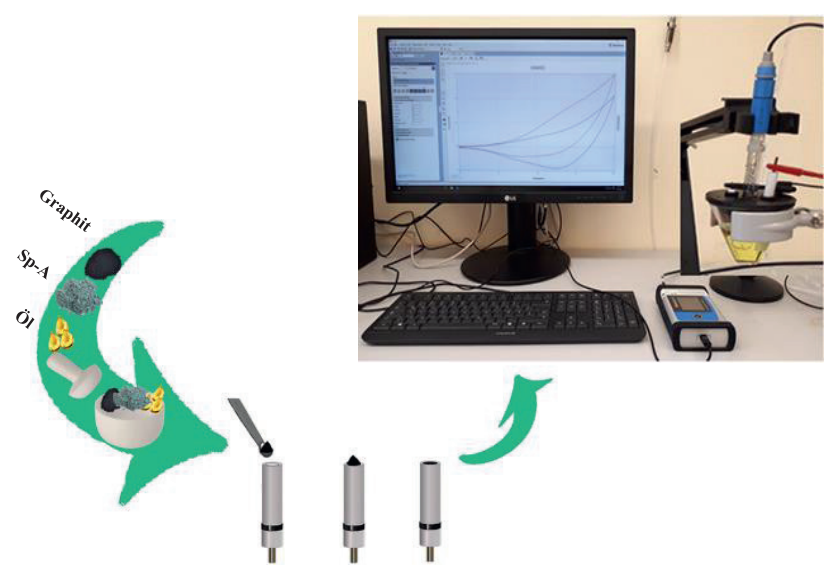

Abb. 1: Schematische Darstellung der Vorbereitung des enzymfreien Sensors und der elektrochemischen Messung. 


\section{Methoden und Materialien}

\section{Herstellung und Charakterisierung des Spongin- Atacamit-Komposits:}

Das Sp. A sowie eine Modell-Kupfer-Ammonium-Lösung wurden nach dem in unserer neueren Arbeit beschriebenen Verfahren hergestellt [20]. Das entwickelte Sp. A wurde bereits mit Hilfe von Röntgenbeugung, Neutrondiffraktion, hochauflösender Transmissionselektronenmikroskopie, Röntgenabsorptions-Feinstrukturspektroskopie an der Nahkante, Röntgen-Photoelektronenspektroskopie und paramagnetischer Elektronenresonanzspektroskopie eingend charakterisiert. Die einzigartige Probe des Atacamit-Mineralstandards (St. A) wurde freundlicherweise von der Mineralogischen Sammlung der TU Bergakademie Freiberg, Deutschland, zur Verfügung gestellt.

\section{Sensor-Herstellung:}

Modifizierte Kohlenstoffpastenelektroden (CPEs) als Sensoren wurden durch Mischen von Graphit und Sp. A-Pulver als Modifikator und dann mit Paraffinöl als Binder in verschiedenen Verhältnissen und Mahldauer gründlich in einem Mörser zu einer homogenen Paste verarbeitet. Alle Komponenten wurden homogenisiert, um eine Mischung zu erhalten, die anschließend in einen Kohlenstoffpastenhalter mit einem Innendurchmesser von $2 \mathrm{~mm}$ gefüllt wurde. Zum Vergleich wurden eine nackte CPE (Bare CPE) und eine mit Standard-Atacamit-Pulver (St. A) modifizierter CPE auf die gleiche Weise hergestellt.

\section{Elektrochemische Messung}

Elektrochemische Untersuchungen wurden mit dem elektrochemischen Analysesystem Palmsens 4 und der herkömmlichen Drei-Elektroden-Konfiguration durchgeführt. Die hergestellten CPEs wurden als Arbeitselektroden verwendet. Eine $\mathrm{Ag} / \mathrm{AgCl}$ (3 $\mathrm{M} \mathrm{KCl}$ )-Elektrode und ein Platindraht dienten als Referenz- bzw. Gegenelektrode.

\section{Ergebnisse}

Um Informationen über den Elektronentransfer zwischen dem Elektrolyten und der Elektrodenoberfläche zu erhalten, wurden die Grenzflächeneigenschaften von blankem CPE und Sp. A/CPE durch elektrochemische Impedanzspektroskopie (EIS) in Gegenwart des Redoxpaars $\mathrm{K}_{3}\left[\mathrm{Fe}(\mathrm{CN})_{6}\right] / \mathrm{K}_{4}\left[\mathrm{Fe}(\mathrm{CN})_{6}\right]$ als elektrochemische Sonde verglichen (Abb. 2).

Zyklische Voltammetriemessungen (CV) wurden zur Evaluierung der katalytischen Leistung der hergestellten Elektro-den für die Glukoseoxidation eingesetzt. Die CVKurven von Bare CPE, Sp.A/CPE und St.A/CPE wurden in $0,1 \mathrm{M} \mathrm{NaOH}$-Lösung ohne und mit $2 \mathrm{mM}$ Glukose aufgenommen (Abb. 3).

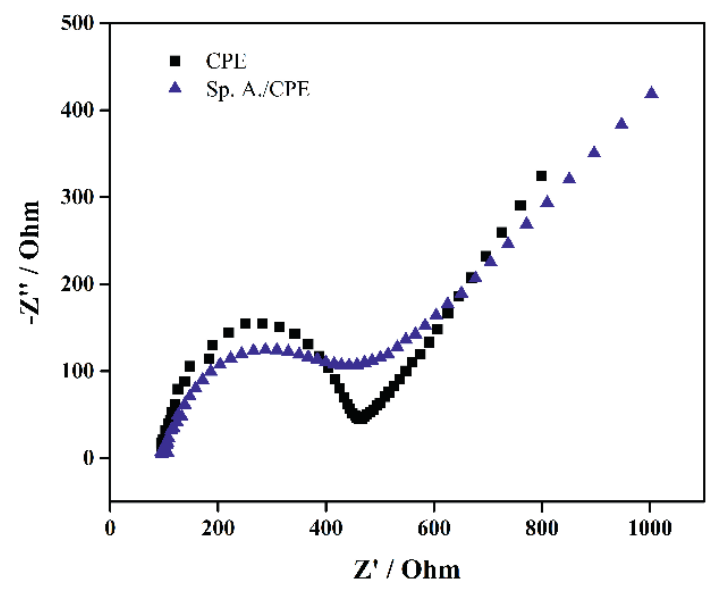

Abb. 2: EIS Ergebnisse von CPE und Sp. A./CPE in einer Lösung von $0.1 \mathrm{M} \mathrm{KCl}$ cmit $5 \mathrm{mM}\left[\mathrm{Fe}(\mathrm{CN})_{6}\right]^{3-/ 4-}$

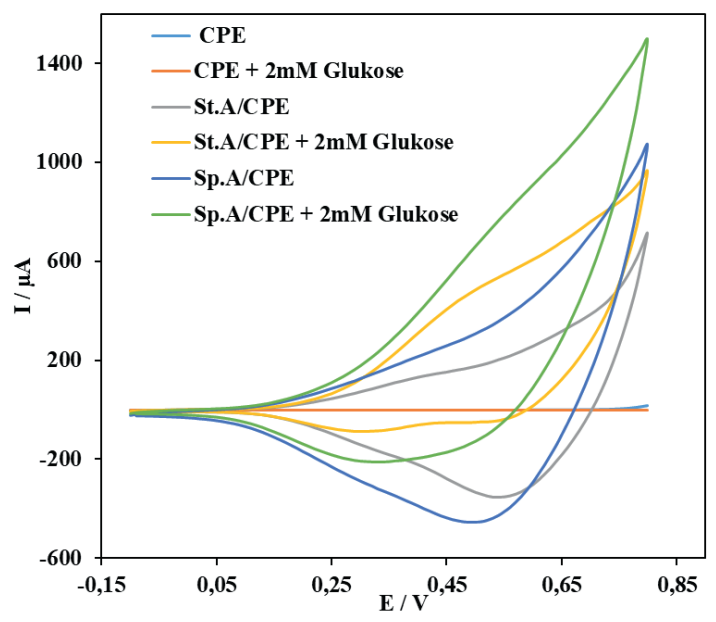

Abb. 3: CVs von CPE, St. A./CPE und Sp. A./CPE in 0,1 $\mathrm{M} \mathrm{NaOH}$-Lösung ohne und mit $2 \mathrm{mM}$ Glukose.

\section{Diskussion}

Wie Abb. 2 zeigt, wies der Bare CPE im Hochfrequenzbereich einen großen Halbkreis mit einem hohen Elektronentransferwiderstand (470 $\Omega$ ) auf, was auf das Vorhandensein von nichtleitendem Paraffinöl zurückzuführen ist. Aber die Sp.A/CPE zeigte einen viel kleineren Elektronenübergangswiderstand, was darauf hindeutet, dass Sp.A ein ausgezeichnetes elektrisch leitendes Material zur Beschleunigung des Elektronentransfers ist.

Wie in Abb. 3 gezeigt, wurde mit der unmodifizierten Eelektrode in Gegenwart von Glukose kein Oxidationsstrom erzielt. Im Vergleich dazu zeigten Sp.A/CPE und St.A/CPE ein deutlich verbessertes Stromverhalten bei der Glukoseoxidation. Wie ersichtlich, zeigte Sp.A/CPE eine ausgezeichnete elektrokatalytische Oxidation (ein breiter Oxidationspeak) von Glukose mit einer geeigneteren elektrochemischen Reaktion als St.A/CPE. Dieses Ergebnis 
deutet darauf hin, dass das 3D-Spongin-Gerüst eine große Oberfläche für Atacamit-Kristalle bietet und so die schnelle Diffusion von Glukosemolekülen im 3D-Netzwerk und den schnellen Elektronentransfer zwischen Glukose und Atacamit fördert. Breite Reduktionspeaks zwischen $+0,45$ und 0,55 V wurden dem $\mathrm{Cu}(\mathrm{II}) / \mathrm{Cu}(\mathrm{III})$-Redoxpaar am Sp.A/CPE bzw. St.A/CPE zugeordnet [13]. Basierend auf früheren Studien ist die Glukoseoxidation stark abhängig von dem Redoxpaar $\mathrm{Cu}(\mathrm{II}) / \mathrm{Cu}(\mathrm{III})$ im alkalischen Medium, das im Potentialbereich von $0,40-0,80 \mathrm{~V}$ auftritt, wo der Oxidationspeak für das $\mathrm{Cu}(\mathrm{II}) / \mathrm{Cu}(\mathrm{III})$-Redoxpaar beobachtet wurde [21-23]. Es wurde vorgeschlagen, dass $\mathrm{Cu}$ (II) zu Cu(III) oxidiert und die erzeugten $\mathrm{Cu}(\mathrm{III})-S p e z i e s$ (wie $\mathrm{CuOOH}$ oder $\left.\mathrm{Cu}(\mathrm{OH})^{4-}\right)$ als aktives Oxidationsmittel für die Oxidation von Glukose zu Gluconolacton wirken und nach der Glukoseoxidation zu Cu(II) reduziert werden. Die Oxidationsstufe des Kupfers an der Oberfläche des Sp.A Komposits ist $\mathrm{Cu}^{2+}$ [20], so dass die Glucose-Elektrooxidation in $\mathrm{NaOH}$ wie folgt erklärt werden kann: während der CV-Messung würde $\mathrm{Cu}(\mathrm{II})$ in den Atacamitkristallen auf der Oberfläche des Sponginskeletts zunächst zu Cu(III) oxidiert. Dann oxidiert $\mathrm{Cu}(\mathrm{III})$ die Glukose zu Gluconolacton, das schließlich weiter zu Gluconsäure hydrolysiert wird.

Um die Reaktionskinetik der Glukoseoxidation auf der Oberfläche von Sp.A/CPE zu untersuchen, wurden CVMessungen bei verschiedenen Scanraten in 0,1 M NaOH mit 2 mM Glukoselösung durchgeführt. Das Resultat zeigte, dass der Oxidationsstrom von Glukose linear proportional zur Quadratwurzel der Sweep-Geschwindigkeiten ansteigt, was darauf hinweist, dass die elektrochemische Oxidation von Glukose auf der Oberfläche von Sp.A/CPE diffusionskontrolliert ist $[5,21]$.

Die amperometrische Antwort des Sp.A/CPE auf eine schrittweise Zugabe von Glucose in $0,1 \mathrm{M} \mathrm{NaOH}$ bei einem optimalen Potential von 0,60 V wurde ebenfalls untersucht. Das Sp.A/CPE zeigte eine ausgezeichnete Aktivität gegenüber Glukose in einem breiten Konzentrationsbereich mit hoher Empfindlichkeit und einer niedrigen Nachweisgrenze. Dies kann auf die große Oberfläche und die gute kristalline Struktur des porösen 3D-Sp.A-Gerüsts zurückgeführt werden, das eine sehr große Anzahl von Interaktionsstellen für elektrochemische Reaktionen bietet. Dies führt zu einer hohen Leistungsfähigkeit des Glukosesensors.

Darüber hinaus ist die Selektivität eines nicht-enzymatischen Glukosesensors aufgrund der Koexistenz verschiedener oxidierbarer Störfaktoren wie Saccharose, Ascorbinsäure, Harnsäure und Dopamin in realen Proben einer der wichtigsten Parameter für den genauen Nachweis von Glukose. Da die normale Glukosekonzentration im Blut oder Plasma höher ist (3,9 und 7,1 mM) als die störende Spezies $(0,1 \mathrm{mM})[24,25]$, wurden die Selektivität und die Anti-Interferenz-Wirkung des vorbereiteten Sp.A/CPE durch sukzessive Zugabe von $1 \mathrm{mM}$ Glukose und anschließende Zugabe von 0,1 mM der genannten störenden Spezies bewertet. Es wurde festgestellt, dass nach der Zugabe von Glukose in die $\mathrm{NaOH}$-Lösung eine deutliche Stromänderung zu beobachten war, während der Strom der störenden Spezies im Vergleich zur Glukosereaktion ungefähr unwesentlichwar.

Um die Leistung der hergestellten Elektrode auf der Basis von Sp.A als praktischer Glukosesensor weiter zu bewerten, wurden andere wichtige Faktoren wie Wiederholbarkeit, Reproduzierbarkeit und Stabilität des Sensors untersucht. Um die Reproduzierbarkeit zu testen, wurden fünf $\mathrm{Sp}-\mathrm{A} / \mathrm{CPEs}$ frisch hergestellt und ihre amperometrischen Reaktionen auf $2 \mathrm{mM}$ Glukose bei +0,6 V untersucht. Die relative Standardabweichung (RSD) betrug 3,56\%, was darauf hindeutet, dass die hergestellten Elektroden sehr gut reproduzierbar sind. Die Wiederholbarkeit von fünf aufeinanderfolgenden Messungen von $2 \mathrm{mM}$ Glukose an einem Sp.A/CPE ergab eine RSD von 2,91\%, was die gute Wiederholbarkeit des hergestellten Sensors bestätigt. Um die Funktionalität des Sensors weiter zu bestätigen, wurde der Langzeitstabilitätstest durch amperometrische Messungen des Sp.A/CPE in Gegenwart von $1 \mathrm{mM}$ Glukose bewertet. Der Strom blieb nach einem Monat bei über $82 \%$ seines ursprünglichen Wertes, was darauf hindeutet, dass der vorgeschlagene Sensor sehr stabil ist. Darüber hinaus wurde die Stromreaktion von Sp.A/CPE in Intervallen von $200 \mathrm{~s}$ nach Injektion von $1 \mathrm{mM}$ Glukose aufgezeichnet. Dabei zeigte sich, dass der amperometrische Strom nach einer Testdauer von $2000 \mathrm{~s}$ immer noch $96 \%$ seines ursprünglichen Wertes betrug.

Als Zusammenfassung haben wir einen hochleistungsfähigen 3D-Komposit auf der Basis des Biopolymers Spongin und Atacamit entwickelt, der erfolgreich als Sensormaterial für die Herstellung eines einfachen, billigen und zuverlässigen nicht-enzymatischen Glukosesensors verwendet werden kann. Es wurden eine hohe Empfindlichkeit, eine gute Selektivität unter verschiedenen Störfaktoren, eine niedrige Nachweisgrenze, breite Linienbereiche mit hervorragender Stabilität sowie eine günstige Wiederholbarkeit und Reproduzierbarkeit erzielt. Diese hervorragende analytische Leistung des Sensors ist auf das einzigartige strukturelle Design des mikroporösen 3D-Spongingerüsts zurückzuführen, das die Aktivität des Atacamits als Elektrokatalysator effizient verbessert. Daher können Glukosemoleküle schnell und einfach in das poröse 3D-Netzwerk diffundieren, was zu einem erleichterten Elektronentransfer zwischen Glukose und Atacamit führt. Da Spongin biotechnologisch kultivierbar ist, kann dieser Sensor als vielversprechender Kandidat für die Entwicklung neuer kommerzieller nicht-enzymatischer Glukosesensoren in großem Maßstab zur Anwendung kommen.

\section{Literatur}

[1] Hassan, M.H., Vyas C., Grieve, B., Bartolo, P., Recent Advances in Enzymatic and Non-Enzymatic Electrochemical Glucose Sensing, Sensors (Basel, Switzerland) 21 (2021).

[2] He, W., Huang, Y., Wu, J., Enzyme-Free Glucose Biosensors Based on MoS2 Nanocomposites, Nanoscale research letters 15 (2020) 60. 
[3] Mishra, A.K., Jarwal, D.K., Mukherjee, B., Kumar, A., Ratan, S., Tripathy, M.R., Jit, S., Au nanoparticles modified $\mathrm{CuO}$ nanowire electrode based non-enzymatic glucose detection with improved linearity, Scientific Reports, (2020) 10:11451.

[4] Wang, L., Jia, S., Wang, N., Cao, X., Atom-diffusion enhanced electrocatalytic activity toward glucose oxidation on atacamite nanorods, Int. J. Nanomanufacturing, (2016) 12:298.

[5] Xu, Z., Wang, Q., Li, R., Zhangsun, H., Dong, M., Wang, L., Surface Selenylation Engineering for Construction of a Hierarchical NiSe2/Carbon Nanorod: A High-Performance Nonenzymatic Glucose Sensor, ACS applied materials \& interfaces 13 (2021) 22866-22873.

[6] Prabhakaran, A., Nayak, P., Surface Engineering of Laser-Scribed Graphene Sensor Enables Non-Enzymatic Glucose Detection in Human Body Fluids, ACS Appl. Nano Mater. 3 (2020) 391-398.

[7] Ye, J.-S., Wen, Y., de Zhang, W., Ming Gan, L., Xu, G.Q., Sheu, F.-S., Nonenzymatic glucose detection using multi-walled carbon nanotube electrodes, Electrochemistry Communications 6 (2004) 66-70.

[8] Chen, M., Cao, X., Chang, K., Xiang, H., Wang, R., A novel electrochemical non-enzymatic glucose sensor based on Au nanoparticle-modified indium tin oxide electrode and boronate affinity, Electrochimica Acta 368 (2021) 137603

[9] Ognjanović, M., Stanković, D.M., Ming, Y., Zhang, H., Jančar, B., Dojčinović, B., Prijović, Ž., Antić, B., Bifunctional ( $\mathrm{Zn}, \mathrm{Fe}) 3 \mathrm{O} 4$ nanoparticles: Tuning their efficiency for potential application in reagentless glucose biosensors and magnetic hyperthermia, Journal of Alloys and Compounds 777 (2019) 454-462.

[10] Liu, Y., Teng, H., Hou, H., You, T., Nonenzymatic glucose sensor based on renewable electrospun Ni nanoparticle-loaded carbon nanofiber paste electrode, Biosensors \& bioelectronics 24 (2009) 3329-3334.

[11] Lu, L.-M., Li, H.-B., Qu, F., Zhang, X.-B., Shen, G.-L., Yu, R.-Q., In situ synthesis of palladium nanoparticlegraphene nanohybrids and their application in nonenzymatic glucose biosensors, Biosensors \& bioelectronics 26 (2011) 3500-3504.

[12] Ridhuan, N.S., Abdul Razak, K., Lockman, Z., Fabrication and Characterization of Glucose Biosensors by Using Hydrothermally Grown ZnO Nanorods, Scientific reports 8 (2018) 13722.

[13] Wang, J., Zhang, W.-D., Fabrication of CuO nanoplatelets for highly sensitive enzyme-free determination of glucose, Electrochimica Acta 56 (2011) 7510-7516.

[14] Wang, J., Thomas, D.F., Chen, A., Nonenzymatic electrochemical glucose sensor based on nanoporous $P t P b$ networks, Anal. Chem. 80 (2008) 997-1004.

[15] Lee, W.-C., Kim, K.-B., Gurudatt, N.G., Hussain, K.K., Choi, C.S., Park, D.-S., Shim, Y.-B., Comparison of enzymatic and non-enzymatic glucose sensors based on hierarchical Au-Ni alloy with conductive polymer, Biosensors \& bioelectronics 130 (2019) 48-54.

[16] Jiang, J., Zhang, P., Liu, Y., Luo, H., A novel non-enzymatic glucose sensor based on a Cu-nanoparticlemodified graphene edge nanoelectrode, Anal. Methods 9 (2017) 2205-2210.

[17] Phetsang, S., Kidkhunthod, P., Chanlek, N., Jakmunee, J., Mungkornasawakul, P., Ounnunkad, K., Copper/reduced graphene oxide film modified electrode for non-enzymatic glucose sensing application, Scientific reports 11 (2021) 9302.

[18] Ehrlich H., Wysokowski M., Żółtowska-Aksamitowska S., Petrenko I., Jesionowski T. Collagens of poriferan origin. Marine Drugs (2018), 16:79.

[19] Jesionowski T., Norman M., Żółtowska-Aksamitowska S., Petrenko I., Yoseph Y., Ehrlich H., Marine spongin: naturally prefabricated $3 D$ scaffold-based biomaterial. Marine Drugs; (2018) 16:88;

[20] Tsurkan D., Simon P., Schimpf C., Motylenko M., Rafaja D., Roth F., Inosov D., Makarova A. A., Stepniak I., Petrenko I., Springer A., Langer E., Kulbakov A., Avdeev M., Stefankiewicz A. R., Heimler K., Kononchuk O., Hippmann S., Kaiser D., Viehweger C., Rogoll A., Voronkina A., Kovalchuk V., Bazhenov V.V., Galli R., Rahimi-Nasrabadi M., Molodtsov S. L., Rahimi P., Falahi S., Joseph Y., Vogt C., Vyalikh D. V., Bertau M., Ehrlich $\mathrm{H}$. Extreme Biomimetics: Designing of the first nanostructured $3 D$ spongin-atacamite composite and its application. Advanced Materials, (2021) 33(30) 2101682.

[21] Sun, S., Sun, Y., Chen, A., Zhang, X., Yang, Z., Nanoporous copper oxide ribbon assembly of free-standing nanoneedles as biosensors for glucose, The Analyst 140 (2015) 5205-5215.

[22] Marioli, J.M., Kuwana, T., Electrochemical characterization of carbohydrate oxidation at copper electrodes, Electrochimica Acta 37 (1992) 1187-1197.

[23] Zhuang, Z., Su, X., Yuan, H., Sun, Q., Xiao, D., Choi, M.M.F., An improved sensitivity non-enzymatic glucose sensor based on a $\mathrm{CuO}$ nanowire modified $\mathrm{Cu}$ electrode, The Analyst 133 (2008) 126-132.

[24] Karikalan, N., Velmurugan, M., Chen, S.-M., Karuppiah, C., Modern Approach to the Synthesis of $\mathrm{Ni}(\mathrm{OH}) 2$ Decorated Sulfur Doped Carbon Nanoparticles for the Nonenzymatic Glucose Sensor, ACS applied materials \& interfaces 8 (2016) 22545-22553.

[25] Chen, Q., Chu, D., Yan, L., Lai, H., Chu, X.-Q., Ge, D., Chen, X., Enhanced non-enzymatic glucose sensing based on porous ZIF-67 hollow nanoprisms, New J. Chem. 45 (2021) 10031-10039. 